\title{
Heat capacity of suspended phonon cavities
}

\author{
A. Gusso ${ }^{1,2 *}$ and Luis G. C. Rego ${ }^{1}$ \\ 1-Departamento de Física, \\ Universidade Federal de Santa Catarina, \\ 88040-900, Florianópolis-SC, Brazil \\ 2-Departamento de Física, \\ Universidade Federal do Paraná, \\ C.P. 19044, 81531-990 Curitiba-PR, Brazil
}

(Dated: March 1, 2018)

\begin{abstract}
We present a detailed analysis of the vibrational spectrum and heat capacity of suspended mesoscopic dielectric plates, for various thickness-to-side ratios at sub-Kelvin temperatures. The vibrational modes of the suspended cavity are accurately obtained from the three-dimensional (3D) elastic equations in the small strain limit and their frequencies assigned to the cavity phonon modes. The calculations demonstrate that the heat capacity of realistic quasi-2D phonon cavities approach the linear dependence on $T$ at sub-Kelvin temperatures. The behavior is more pronounced for the thinnest cavities, but takes place also for moderately thick structures, with thickness-to-side ratios $\gamma=0.1$ to 0.2 . It is also demonstrated that the heat capacity of the suspended phonon cavities is invariant under the product of the temperature $(\mathrm{T})$ with a characteristic lateral dimension $(\mathrm{L})$ of the sample. The present results establish a lower bound for the heat capacity of suspended mesoscopic structures and indicate the emergence of the quantum mechanical regime in the dynamics of bounded phonon cavities.
\end{abstract}

PACS numbers: $85.85 .+\mathrm{j}, 63.22 .+\mathrm{m}, 63.70 .+\mathrm{h}$

\section{INTRODUCTION}

Suspended nanostructures have become relevant elements for both basic research and technology. Microelectromechanical systems (MEMS), such as cantilevers, gears and membranes, already find widespread use in several technological applications ${ }^{1}$. At the same time, current developments in surface nanomachining render possible the controlled fabrication of a large variety of suspended nanostructures 2.3 having, in particular, an extremely weak thermal coupling with the environment. As a consequence, ultrasensitive bolometers $\underline{\underline{4}}$ and calorimeters 5.6 have been developed with unprecedented sub-attojoule resolution, for operation in the $T \lesssim 5$ Kelvin temperature range, envisaging the possibility of measuring the heat capacity of nano-objects and, eventually, even single molecules ${ }^{7}$. In the realm of fundamental research, the operation of nanoelectromechanical structures (NEMS) is finally approaching the quantum regime $e^{8,9,10}$. The construction of suspended solid state quantum logic gates 11.12 is amid the applications anticipated for such structures, since the electron-phonon interaction, which is a source of decoherence and dissipation for both quantum dot qubits $\underline{13,14}$ and single-electron transistors (SET) $)^{15}$, can be controlled in them $16,17,18,19$.

In fact, for most of the cases mentioned above the devices are operated at sub-Kelvin temperatures; the requirement of ultracold temperatures being specially severe for the operation of quantum logic gates. For in-

\footnotetext{
*Present address: Depto. de Ciências Exatas e Tecnológicas, Universidade Estadual de Santa Cruz, CEP 45662-000, Ilhéus-BA
}

stance, in recent experimental realization, quantum dot charge-qubits $\frac{13,14}{}$ and suspended SET 15 have been operated at a base temperature of $20 \mathrm{mK}$. It is therefore reasonable to expect that suspended nanostructures comprising such quantum devices as well as ultrasensitive bolometers and calorimeters will be functioning at temperatures $T \lesssim 1 \mathrm{~K}$.

Despite the interest, there is not yet a comprehensive theory for the electron-phonon interaction in suspended nanostructures ${ }^{20,21}$. A central issue of the problem is the difficulty of rigorously describing the low temperature acoustic phonon spectrum in suspended nano-devices, which is fundamental for determining (i) the electronphonon interaction with its many consequences for the device operation and (ii) the device's thermal properties, such as its thermal conductivity and heat capacity. At sub-Kelvin temperatures, the formalisms adopted for bulk materials may not produce correct results for the phonon spectrum of suspended nanostructures because the wavelength and mean free path of the dominant phonons can be bigger than the physical dimensions of the structure. Moreover, there are no general analytical solutions for the vibrational modes of bounded suspended plates $^{22}$.

Motivated by these circumstances this work presents a detailed study of the phonon spectrum and the heat capacity $\left(C_{V}\right)$ of suspended rectangular dielectric nanostructures of various thicknesses at sub-Kelvin temperatures. The vibrational modes of the suspended cavity are accurately calculated from the three-dimensional (3D) elastic equations in the small strain limit and the obtained frequencies assigned to the cavity phonon modes. After obtaining a reliable phonon spectrum, with convergence assured for a few thousand cavity modes, the heat 
capacity of isolated suspended mesoscopic phonon cavities having $3 \mathrm{D}$ and quasi-2D character is investigated. For such systems, the calculations demonstrate that the temperature dependence of $C_{V}$ approaches the linear regime in sub-Kelvin temperatures, the effect being more pronounced for quasi-2D nanostructures. Nonetheless, a simple model of plane waves yields a phonon spectrum in good agreement with the 3D elastic model for the very thick suspended nanostructures. A dimensional analysis of the free vibrational modes also reveal that the heat capacity of the rectangular phonon cavities has the scale invariant form, that is, $C_{V}$ is invariant with respect to the product of the temperature with a characteristic lateral dimension. The present results indicate that the low temperature heat capacity of quasi-2D suspended nanostructures may have been underestimated and, therefore, sets a lower bound for their heat capacity.

\section{THEORETICAL FORMULATION}

We consider suspended nanostructures of rectangular geometry, with lateral dimensions defined by $L_{x}$ and $L_{y}$, and thickness $L_{z}$. Such a choice is motivated by the fact that several recent experiments, 23,24 , probing thermal and electrical properties of suspended nanostructures, have utilized square or rectangular plates with thicknessto-side ratios $\left(\gamma=L_{z} / L_{y}\right)$ including 0.1 [23], 0.04 [24], and 0.015 [4]. The first structure is considered to be a moderately thick plate, while the last two cases are examples of thin plates. Because the suspended nanostructures are usually made of non-crystalline materials, like poly-silicon and amorphous SiN (Silicon Nitride), in this work the phonon cavities are taken to be homogeneous and isotropic rectangular structures.

Because at sub-Kelvin temperatures the dominant phonon modes are long wavelength acoustic ones, with a mean free path that exceeds the dimensions of the structure $\frac{4,23,24}{2}$, we resort to the elasticity theory to obtain the phonon spectrum of the suspended nanostructures. In this limit the phonons correspond to the free vibrational modes of the cavity $\underline{18}$, as determined by the elastic theory of solids 25 . The continuum elasticity model has been successfully used to describe the properties of propagating phonons in beams ${ }^{26,27}$, thin membranes ${ }^{16.28}$, and arrays of nanomechanical resonators ${ }^{29.30}$.

To secure the correct description of the thermal properties of suspended nanostructures of a few $\mu m^{2}$ in area in the sub-Kelvin temperature regime, at least a few thousand vibrational modes have to be calculated with confidence. A variety of methods intended to calculate the free vibrations of thick plates have been developed 22 . Since the simplified models like the Classical Plate Theory $(\mathrm{CPT})^{31}$ are adequate only for the lowest modes of thin plates, a three-dimensional analysis of the free vibrations of the cavity is necessary. In general such methods utilize the Rayleigh-Ritz formalism to determine the displacement field, which is represented as a series of or- thogonal polynomials 32,33 . In this work we follow the procedure developed by Zhou et al ${ }^{32}$ due to its simplicity and generality as well as for producing very accurate natural frequencies. For the sake of completness, the method is summarized next.

For the problem of the free vibrations of isotropic structures in the small strain approximation, the kinetic $(\mathrm{T})$ and strain $(\mathrm{U})$ elastic energy functionals for the displacement field $\mathbf{u}(\vec{r}, t)=\mathbf{U}(\vec{r}) e^{i \omega t}$ can be written as

$$
\begin{aligned}
& \mathbf{T}=\frac{\rho}{2} \int\left[\left(\frac{\partial u_{x}}{\partial t}\right)^{2}+\left(\frac{\partial u_{y}}{\partial t}\right)^{2}+\left(\frac{\partial u_{z}}{\partial t}\right)^{2}\right] d v \\
& \mathbf{U}=\frac{E}{2(1+\nu)} \int\left(\frac{\nu \Lambda_{1}^{2}}{1-2 \nu}+\Lambda_{2}+\frac{\Lambda_{3}}{2}\right) d v
\end{aligned}
$$

where $\rho$ is the mass density, $E$ is the Young's modulus and $\nu$ the Poisson's ratio. The $\Lambda$ quantities in the strain energy term are: $\Lambda_{1}=\sum_{i} \varepsilon_{i i}, \Lambda_{2}=\sum_{i} \varepsilon_{i i}^{2}$ and $\Lambda_{3}=\sum_{i<j} \varepsilon_{i j}$, for $i, j=(x, y, z)$, with the components of the strain $\varepsilon_{i i}=\partial_{i} u_{i}$ and $\varepsilon_{i j}=\partial_{j} u_{i}+\partial_{i} u_{j}$. It is convenient to normalize the coordinates with respect to the dimensions of the plate, defining the dimensionless variables $\xi=2 x / L_{x}, \eta=2 y / L_{y}$ and $\zeta=2 z / L_{z}$ in the interval $[-1,1]$. The time-independent displacement field $\mathbf{U}(\vec{r})$ is then written as a sum of orthogonal Chebyshev polynomials multiplied by boundary functions $F_{\delta}(\xi, \eta)$

$$
\begin{aligned}
& U_{\mathrm{x}}(\xi, \eta, \zeta)=F_{\mathrm{x}}(\xi, \eta) \sum_{i, j, k} A_{i j k} P_{i}(\xi) P_{j}(\eta) P_{k}(\zeta) \\
& U_{\mathrm{y}}(\xi, \eta, \zeta)=F_{\mathrm{y}}(\xi, \eta) \sum_{i, j, k} B_{i j k} P_{i}(\xi) P_{j}(\eta) P_{k}(\zeta) \\
& U_{\mathrm{z}}(\xi, \eta, \zeta)=F_{\mathrm{z}}(\xi, \eta) \sum_{i, j, k} C_{i j k} P_{i}(\xi) P_{j}(\eta) P_{k}(\zeta)
\end{aligned}
$$

with the summations beginning from zero. The functions $P_{n}(\chi)$ are Chebyshev polynomials of the first kind and degree $n$, defined by the relation

$$
P_{n}(\chi)=\cos [n \arccos (\chi)],
$$

with $n$ a non-negative integer. The boundary functions $F_{\mathrm{x}}(\xi, \eta), F_{\mathrm{y}}(\xi, \eta)$ and $F_{\mathrm{z}}(\xi, \eta)$ have the general form $F_{\delta}(\xi, \eta)=f_{\delta}^{1}(\xi) f_{\delta}^{2}(\eta)$, with $\delta=\mathrm{x}, \mathrm{y}, \mathrm{z}$. For our purposes the boundary conditions of interest are FF (freefree), CC (clamped-clamped) and $\mathrm{CF} / \mathrm{FC}$, which correspond, respectively, to the functions $f_{\delta}^{1}(\xi) \equiv f_{\delta}^{2}(\eta)=1$, $f_{\delta}^{1}(\xi) \equiv f_{\delta}^{2}(\eta)=1-\eta^{2}$, and $f_{\delta}^{1}(\xi) \equiv f_{\delta}^{2}(\eta)=1 \pm \eta$.

Substituting the series representation for $\mathbf{u}(\vec{r}, t)=$ $\mathbf{U}(\vec{r}) e^{i \omega t}$ into Eqs. (11) and (2), one obtains $\mathrm{T}_{\max }$ and $\mathrm{U}_{\text {max }}$, which are the maximum values of $\mathrm{T}$ and $\mathrm{U}$ during a vibratory cycle. The frequency determinant is formulated by minimizing the functional $\mathrm{U}_{\max }-\mathrm{T}_{\max }$ with respect to each of the coefficients $\{A\},\{B\}$ and $\{C\}$, to produce the $3 \mathrm{D}$ elastic equations of motion

$$
\left([K]-\Omega^{2}[M]\right)\left(\begin{array}{l}
\{A\} \\
\{B\} \\
\{C\}
\end{array}\right)=0,
$$


TABLE I: Physical constants of relevant materials in the amorphous phase.

\begin{tabular}{crcc}
\hline \hline Material & $\rho\left(\mathrm{g} / \mathrm{cm}^{3}\right)$ & $E(\mathrm{GPa})$ & $\nu$ \\
$\mathrm{GaAs}^{34}$ & 5.1 & 71 & 0.32 \\
$\mathrm{Si}{ }^{35}$ & 2.3 & 170 & 0.22 \\
$\mathrm{SiN} 36$ & 3.1 & 285 & 0.20 \\
$\mathrm{SiC}{ }^{37}$ & 3.0 & 400 & 0.20 \\
\hline \hline
\end{tabular}

where $\Omega=\omega L_{x} \sqrt{\rho / E}$ is a dimensionless parameter and $\omega$ is the free vibration frequency, to be assigned to the phonons. In Eq. (7), $[K]$ and $[M]$ denote the symmetric stiffness matrix and the block diagonal mass matrix, respectively, which can be found in explicit form in Ref. [32]. Another useful dimensionless parameter associated with the frequency is $\Delta=\left(\Omega / \lambda \gamma \pi^{2}\right) \sqrt{12\left(1-\nu^{2}\right)}$, with $\lambda=L_{x} / L_{y}$ and $\gamma=L_{z} / L_{y}$, which yields the normalized frequencies for the family of all rectangular plates with the same aspect ratios $(\lambda$ and $\gamma)$ and elastic constants $(E$ and $\nu)$.

An important aspect of the present analysis is the reliability of the phonon spectrum to be used in the evaluation of the thermodynamical properties of the nanostructure. For that reason the convergence of the highest frequencies was limited to be within $5 \%$. The spectrum span can be extended by increasing the amount of basis functions $P_{n}(\chi)$ used in the representation of $\mathbf{U}(\vec{r})$. In the instance of the thickest square plate to be considered, with $\gamma=0.5$, we have used $n_{x}=n_{y}=29$ and $n_{z}=13$ Chebyshev polynomials, yielding approximately 12000 reliable modes. For the thinnest plate, $\gamma=0.02$, the best results were obtained for $n_{x}=n_{y}=51$ and $n_{z}=4$ that yielded 4000 reliable phonon modes. The number of reliable frequency modes will determine the maximum temperature $\left(T_{\max }\right)$ for which the heat capacity can be calculated with confidence.

The dependence of the phonon frequencies on the material parameters is such that higher frequencies are obtained for stiff and light materials. Table \ contains the values of the mass density, Young's modulus and Poisson's ratio for materials of relevance for the fabrication of NEMS. This work investigates nanostructures made of amorphous silicon carbide (a-SiC) because of its high rigidity and widespread use in the fabrication of suspended NEMS.

The calculated spectra exhibit significant dependence on the dimensions of the nanostructure, as illustrated in Figure 1 for the first 2500 vibrational modes of free standing a-SiC mesoscopic structures. The structures have the same lateral dimensions $L_{x}=L_{y}=L=2 \mu \mathrm{m}$ but different thickness-to-side ratios: $\gamma=0.02$ (solid), 0.05 (dashed) and 0.1 (dot-dashed). At the lower part of the spectrum the frequencies are higher for the thick plates, however, the behavior is reversed as the mode index $\alpha$ increases. The frequencies are also inversely proportional to the area of the plate. Moreover, by a numerical analysis of the eigenfrequencies it was observed that the vibrational spectrum of the cavities can be very well described by the form $\omega=\omega_{0} \alpha^{\phi}$ in two limiting cases: for the quasi-2D phonon cavities $(\gamma \leq 0.02)$ the fitting yields $\phi \lesssim 1$, whereas for the three-dimensional (thick) phonon cavities $(\gamma>0.2)$ one obtains $0.4<\phi<0.5$. Between the two cases, i.e. for moderately thick nanostructures, the frequencies cannot be well described by a single power curve.

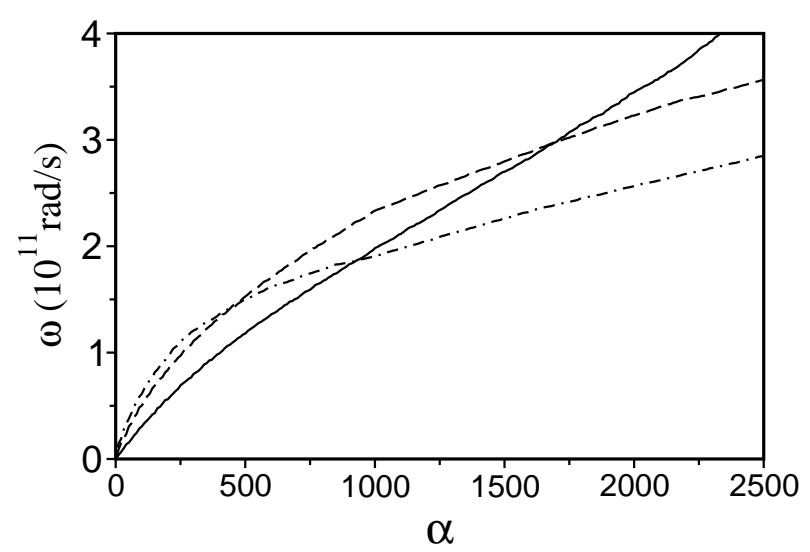

FIG. 1: Natural vibration frequencies, as a function of the mode index $\alpha$, calculated by the 3D method for a free standing square $(\lambda=1)$ a-SiC nanostructure of sides $L=2 \mu \mathrm{m}$ and $\gamma=L_{z} / L$ equal to 0.02 (solid), 0.05 (dashed) and 0.1 (dotdashed).

In the following we examine the heat capacity of the phonon cavities, as predicted by the $3 \mathrm{D}$ analysis. For the sake of comparison, we also utilize a basic model to describe the confined phonons, which comprises some of the reductionist features commonly found in the literature ${ }^{7.38}$. Its main assumption is that the phonons can be described by plane waves with three independent polarizations: one longitudinal and two transverse. In addition, as the dimensions of the structure become sufficiently small, i.e. comparable to the mean free path of the phonons, these become standing waves satisfying the appropriate boundary conditions. The method is here designated Bounded Plane Wave Model (BPWM). For the nanostructures under consideration and because of the very low temperature, it is assumed that the phonons form standing waves in all three directions. In comparison with the $3 \mathrm{D}$ analysis, different predictions for the specific heat are expected on the basis of the BPWM, owing to its naive representation of the phonon modes in suspended nanostructures. However, despite the simplicity, it will be shown that the BPWM can describe the heat capacity of thick phonon cavities quite well.

According to the BPWM, the phonon spectrum of a freely suspended nanostructure is easily obtained from 
the wave vectors

$$
\kappa_{l m n}^{2}=\pi^{2}\left[\left(\frac{l}{L_{x}}\right)^{2}+\left(\frac{m}{L_{y}}\right)^{2}+\left(\frac{n}{L_{z}}\right)^{2}\right],
$$

with $l, m$ and $n$ integers. The frequencies for the longitudinal and transverse modes are given by $\omega_{l m n}^{l}=v^{l} \kappa_{l m n}$ and $\omega_{l m n}^{t}=v^{t} \kappa_{l m n}$, with the sound velocities of the a$\mathrm{SiC}$ obtained from the elastic constants of the material: $v^{l}=12,170 \mathrm{~m} / \mathrm{s}$ and $v^{t}=7,450 \mathrm{~m} / \mathrm{s}$.

\section{HEAT CAPACITY OF SUSPENDED PHONON CAVITIES}

Having calculated the displacement modes $\mathbf{U}_{\alpha}(\vec{r})$ and the associated eigenfrequencies $\omega_{\alpha}$, corresponding to the free vibrations of the plate, the quantum mechanical phonon modes of the cavity are obtained by the standard quantization procedure $\frac{18}{}$. As a result, we ascribe the energy $\mathcal{U}=\sum_{\alpha}\left(n_{\alpha}+1 / 2\right) \hbar \omega_{\alpha}$ to the phonon system and calculate the quantum mechanical heat capacity of the phonon cavity as

$$
C_{V}(T)=\frac{\partial \mathcal{U}}{\partial T}=\frac{\partial}{\partial T} \sum_{\alpha} \frac{\hbar \omega_{\alpha}}{\exp \left(\hbar \omega_{\alpha} / k_{B} T\right)-1}
$$

with $n_{\alpha}$ given by Planck's distribution. Since the harmonic regime complies with the small strain limit that is assumed in the present derivations, the constant-volume specific heat $\left(c_{v}\right)$ must equal the constant-pressure specific heat $\left(c_{p}\right)^{39}$. Moreover, for bulk metallic samples it is generally found that the low temperature specific heat varies as $c_{v}=A T+B T^{3}$, comprising the electron and phonon contributions, respectively.

Next we show predictions for the heat capacity obtained through the 3D analysis as well as results gained by the simplified method. The phonon cavities to be considered are free standing a-SiC square plates $(\lambda=1)$ with lateral dimensions $L=2 \mu m$ and having different thickness-to-side ratios, namely $\gamma=0.02,0.05,0.1,0.2$ and 0.5. $C_{V}$ is calculated for temperatures $T \leqslant T_{\max }$, where $T_{\max }$ is the maximum temperature that allows reliable results to be obtained with the available phonon modes. That is, if $T>T_{\max }$ additional modes must be included in the calculation of $C_{V}$ since the occupation of the high energy modes is increases. Figure 2(a) presents the specific heat $c_{v}=C_{V} / V$ as a function of temperature for phonon cavities of different thicknesses, as obtained through the $3 \mathrm{D}$ analysis. For temperatures $T \lesssim 100$ $\mathrm{mK}$ the calculations reveal more than an order of magnitude difference between the quasi-2D $(\gamma \leqslant 0.05)$ and the three-dimensional $(\gamma \gtrsim 0.2)$ phonon cavities. Figure 2 (b) shows the heat capacity $\left(C_{V}(T)\right)$ for two limiting cases, represented by the quasi-2D $(\gamma=0.02)$ and fully three-dimensional $(\gamma=0.5)$ suspended nanostructures. Results gained from the BPWM are also shown by the gray curves. Particularly, the BPWM predicts very good results for the three-dimensional cavities, but seriously
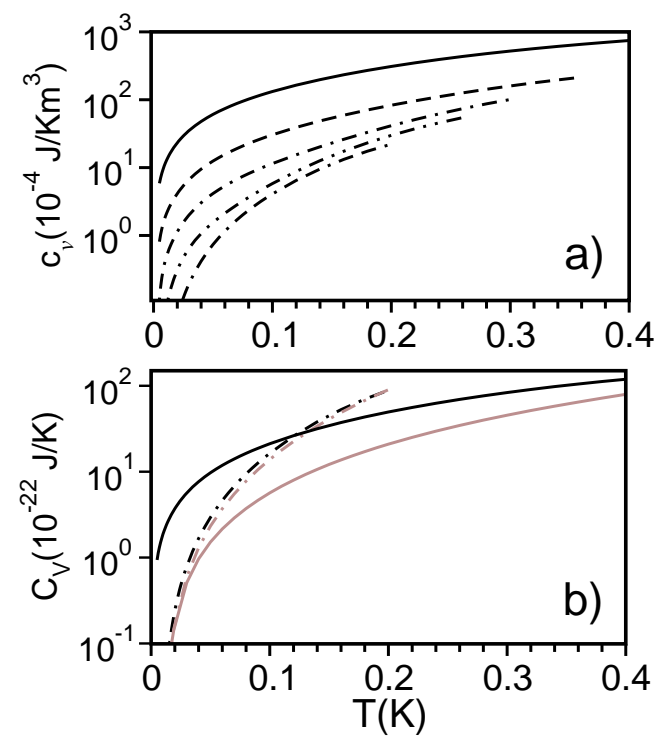

FIG. 2: a) Specific heat $\left(c_{v}=C_{V} / V\right)$ as a function of temperature obtained from the $3 \mathrm{D}$ analysis for a free standing a-SiC square cavity of lateral dimensions $L=2 \mu \mathrm{m}$ and $\gamma=0.02$, $0.05,0.1,0.2$ and 0.5 , in that order from top to bottom. b) The heat capacity $\left(C_{V}\right)$ for the quasi-2D $\gamma=0.02$ (solid) and fully $3 \mathrm{D} \gamma=0.5$ (dashed). The gray curves are predictions from the BPWM (refer to the text).

underestimates the heat capacity of the quasi-2D structures.

Throughout the analysis we have considered thin as well as thick suspended nanostructures. That raises the question, how is the system's dimensionality reflected on the behavior of $C_{V}(T)$ ? In Ref. [38] the dependence of the specific heat on the dimensionality of the system was investigated with a model similar to the BPWM. It was shown that the relation $C_{V} \propto T^{d}$ should hold for a confined phonon gas in the low temperature limit, with $d$ as the system's dimensionality, supporting the inaccurate notion that $C_{V} \propto T^{2}$ for quasi-2D phonon cavities at sub-Kelvin temperatures. Here we perform such an analysis and demonstrate instead that the heat capacity of realistic quasi-2D phonon cavities approaches the linear dependence $C_{V} \propto T$ in the low temperature limit. For that purpose consider the quantity

$$
p_{C}(T)=T \frac{\partial\left(\ln C_{V}(T)\right)}{\partial T},
$$

which provides the temperature dependence of $C_{V}(T)$. For instance, if the heat capacity is given by $C_{V} \propto T^{\alpha}$, we have simply $p_{C}=\alpha$.

Figure 3presents the calculated values of $p_{C}(T)$ for free standing square plates with lateral dimensions $L=2 \mu \mathrm{m}$ and the thickness-to-side ratios previously considered, ranging through the quasi-2D to the fully $3 \mathrm{D}$ cases. The upper panel shows that $C_{V}$ approaches the linear $C_{V} \propto T$ behavior at temperatures $T \lesssim 0.3 K$, particularly in the case of the thinnest cavities with $\gamma=0.02$ and 0.05. Such 


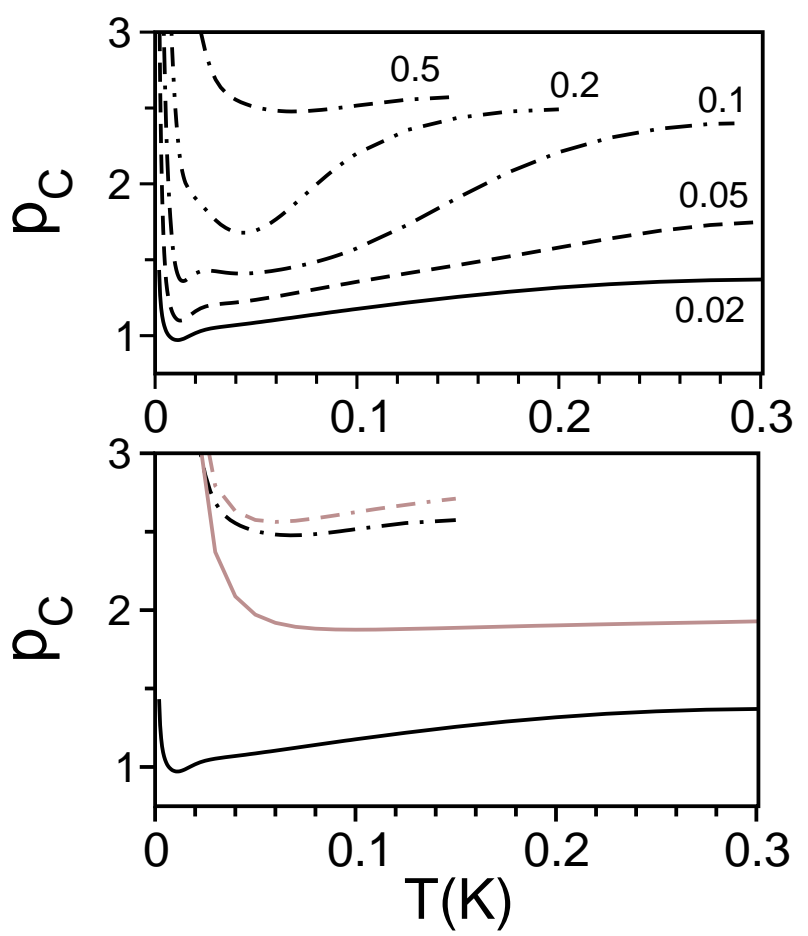

FIG. 3: $p_{C}(T)$ for free standing a-SiC square plates with $L=$ $2 \mu \mathrm{m}$. In the upper panel the predictions of the 3D analysis for different $\gamma$, as indicated by the labels. In the lower panel $p_{C}$ for the quasi-2D $(\gamma=0.02)$ and fully $3 \mathrm{D}(\gamma=0.5)$ cases. The gray curves are the results gained from the BPWM.

a result is expected to hold for strict 2D systems like graphene, although the effect has been predicted also for the specific heat of a $2 \mathrm{D}$ array of nanomechanical resonators 29 . In fact, the sub- $T^{2}$ behavior is observed here even in the case of the moderately thick cavities with $\gamma=0.1$ and 0.2 , at lower temperatures. As the temperature increases $p_{C}$ tends to 3 , indicating that the thickness of the cavity becomes much larger than the dominant phonon wavelength; on the other hand, for vanishing small temperatures, lower than the fundamental vibrational energies, the phonon cavity behaves as a $0 \mathrm{D}$ system. In this case the specific heat decreases exponentially and $p_{C}$ diverges with $T^{-1}$. The $C_{V} \propto T$ behavior is commonly associated with quasi-1D systems like single-wall nanotubes (SWNT) at low temperatures 40 . However, as the temperature decreases beyond $T \lesssim 0.5 K$ a sublinear behavior is observed $n$ such systems $\stackrel{41}{ }$. The effect is ascribed to the overwhelming contribution of the flexural modes to $C_{V}$, since those modes present $\omega(q) \propto q^{2}$ and consequently $C_{V} \propto T^{1 / 2}[42,43]$.

The lower panel of Figure 3 compares the results obtained from the 3D analysis with those of the simple BPWM. According to earlier calculations, both methods produce similar results for fully three-dimensional structures, but the BPWM yields the wrong $p_{C} \approx 2$ value for quasi-2D structures. In the last case, it has been verified that the simple classical plate theory (CPT) for flexu- ral modes, which reduces the dimensions of the problem from three to two by incorporating some of the plate's characteristics such as bending moments 25,31 , yields a close estimate for the temperature dependence of $C_{V}(T)$. The CPT fails, however, as the temperature raises above $T \gtrsim \hbar \pi v_{s} /\left(L_{z} k_{B}\right), v_{s}$ being the sound velocity, because longitudinal and torsional modes begin to contribute significantly to $C_{V}$. Because of the significant difference between the predictions made by the 3D analysis and the BPWM for thin nanostructures, $p_{C}$ may be a convenient observable to experimentally determine the emergence of coherent quantum mechanical dynamics in mesoscopic phonon cavities.

An additional property of the suspended phonon cavities is the scale invariant character of their heat capacity, described as $C_{V}=\mathcal{F}(T L)$, where $\mathcal{F}$ represents the functional in Eq. (9). Namely, $C_{V}$ is invariant regarding the product of the temperature $(T)$ with a characteristic lateral dimension $(L)$ of the structure. For the sake of clarity we consider a square cavity, but the same result can be derived for rectangular cavities with $L_{x}=\lambda L_{y}$, or triangular ones. First notice that the phonon frequency can be written as

$$
\omega_{\alpha}=\frac{\pi^{2} \gamma}{L} \Delta_{\alpha} \sqrt{\frac{E}{12 \rho\left(1-\nu^{2}\right)}} .
$$

Thus $\omega_{\alpha} \propto 1 / L$, for plates of a given thickness-to-side ratio $\gamma$. The dimensionless parameter $\Delta_{\alpha}$ is also a function of $\gamma$ and $\lambda$, therefore independent of the absolute dimensions of the plate. Then, from the definition of the heat capacity, Eq. (9), with the derivative and summation operations commuted, it is easily verified that a transformation that leaves $\omega_{\alpha} / T \propto 1 /(L T)$ invariant does not change the heat capacity. Consequently, the results that have been presented for square phonon cavities of lateral dimension $L=2 \mu \mathrm{m}$ can be generalized for congruent cavities of arbitrary size $L^{\prime}$, with the temperature re-scaled to $T^{\prime}=\left(L / L^{\prime}\right) T$.

Different types of suspended cavities were also investigated, such as bridges (CCFF) and cantilever-like (CFFF) structures, yielding results in qualitative agreement with those previously illustrated, for both the vibrational spectrum and the specific heat. It is observed that the parameter $p_{C}$ shows a tendency towards the value 1 for elongated structures. For instance, in the case of $\gamma=0.1$ and $\lambda=L_{x} / L_{y}=4$ we obtained $p_{c} \approx 1.5$ at $T=T_{\max }$.

The hitherto calculations of the heat capacity of phonon cavities have not taken into account the additional degrees of freedom comprised by impurities, disorder and surface defects, etc. that will be responsible for an increase of $C_{V}$. In particular, the specific heat of bulk noncrystalline solids exhibits an anomalous linear variation with the temperature $\underline{\underline{4}}$ for $T<1 \mathrm{~K}$. The present results, however, set a lower bound for the specific heat of such dielectric nanostructures. 


\section{CONCLUSIONS}

We presented a detailed investigation of the vibrational spectrum and the heat capacity of suspended dielectric mesoscopic structures of various thicknesses at subKelvin temperatures. More than 4000 frequency modes of the cavity were accurately obtained from the 3D elastic equations in the small strain regime. It is therefore demonstrated that the low temperature heat capacity of realistic quasi-2D phonon cavities have an approximate linear dependence on $T$, a result that contradicts estimates obtained by simple models. The sub- $T^{2}$ variation of the heat capacity is observed even for the moderately thick mesoscopic structures. The results show the importance of a fully 3D analysis based on the elastic equa- tions of suspended plates, bridges and cantilevers, for the correct determination of their thermal properties. Finally, the sub- $T^{2}$ effect evidences the quantum mechanical nature of the phonon cavity dynamics and sets a lower bound for their specific heat. The reported results should have special interest for suspended nanostructures intended to be part of solid state quantum devices.

\section{ACKNOWLEDGMENTS}

The authors acknowledge financial support from $\mathrm{CNPq} /$ Brasil and funding provided by Projeto Universal - CNPq. We thank W. Figueiredo and M.E.G. da Luz for comments and suggestions.
1 B. Bushan (editor), Springer Handbook of Nanotechnology (Springer, Berlin,2004).

2 A. N. Cleland, Foundations of Nanomechanics (SpringerVerlag, 2002).

3 K.L. Ekinci and M.L. Roukes, Rev. Sc. Inst. 76, 061101 (2005).

4 C. S. Yung, D. R. Schmidt, and A. N. Cleland, Appl. Phys. Lett. 81, 31 (2002).

5 W. Chung Fon, Keith. C. Schwab, John M. Worlock, and Michael L. Roukes, Nano Lett. 5, 1968 (2005).

6 O. Bourgeois, S.E. Skipetrov, F. Ong, and J. Chaussy, Phys. Rev. Lett. 94, 057007 (2005).

7 M. L. Roukes, Physica B 263-264, 1 (1999).

8 R.G. Knobel and A.N. Cleland, Nature 424, 291 (2003).

9 M.D. LaHaye, O. Buu, B. Camarota, K.C. Schwab, Science 304, 74 (2004).

10 M. Blencowe, Phys. Rep. 395, 159 (2004).

11 A. D. Armour, M. P. Blencowe, and K. C. Schwab, Phys. Rev. Lett. 88, 148301 (2002).

12 A.N. Cleland and M.R. Geller, Phys. Rev. Lett. 93, 070501 (2004).

13 T. Hayashi, T. Fujisawa, H.D. Cheong, Y.H. Jeong, and Y. Hirayama, Phys. Rev. Lett. 91, 226804 (2003); T. Fujisawa, T. Hayashi and Y. Hirayama, J. Vac. Sci. Tech. B 22, 2035 (2004).

14 J. Gorman, E.G. Emiroglu, D. G. Hasko, and D. A. Williams, Phys. Rev. Lett. 95, 090502 (2005).

15 E.M. Weig et al., Phys. Rev. Lett. 92, 046804 (2004).

16 S. Debald, T. Brandes, and B. Kramer, Phys. Rev. B 66, 041301(R) (2002).

17 L. G. C. Rego, A. Gusso, and M. G. E. da Luz, J. Phys. A: Math. Gen. 38, L639 (2005).

18 A. Gusso, M. G. E. da Luz, and L. G. C. Rego, Phys. Rev. B 73, 035436 (2006).

19 B.A. Glavin, V.I. Pipa, V.V. Mitin, and M.A. Stroscio, Phys. Rev.B 65, 205315 (2002).

${ }^{20}$ W. Fon, K.C. Schwab, J.M. Worlock, and M.L.Roukes, Phys. Rev. B 66, 045302, (2002); S. Barman and G.P. Srivastava, Phys. Rev. B 73, 205308 (2006).

21 S.X. Qu, A.N. Cleland, and M.R. Geller, Phys. Rev. B 72, 224301 (2005).

22 A comprehensive literature review of the problem can be found in: L.M. Liew, Y. Xiang, and S. Kitipornchai, J.
Sound and Vib. 180(1), 163 (1995).

23 T. S. Tighe, J. M. Worlock, and M. L. Roukes, Appl. Phys. Lett. 70, 2687 (1997).

${ }^{24}$ K. Schwab et al., Nature 404, 974 (2000).

${ }^{25}$ Karl F. Graff, Wave Motion in Elastic Solids, (Dover, NY, 1975).

${ }^{26}$ L. G. C. Rego and G. Kirczenow, Phys. Rev. Lett. 81, 232 (1998).

27 D.H. Santamore and M.C. Cross, Phys. Rev. B 66, 144302 (2002)

28 T. Kühn, D.V. Anghel, J.P. Pekola, M. Manninen, and Y.M. Galperin, Phys. Rev. B 70, 125425 (2004).

29 D.M. Photiadis, J.A. Bucaro, and X. Liu, Phys. Rev. B 73, 165314 (2006).

30 M.K. Zalalutdinov et al., Appl. Phys. Lett. 88, 143504 (2006).

31 G. F. Elsbernd and A. W. Leissa, Developments in Theoretical and Applied Mechanics 4, 19 (1970).

32 D. Zhou, Y.K. Cheung, F.T.K. Au, and S.H. Lo, Int. J. of Solids Structures 39, 6339 (2002).

33 K.M. Liew, K.C. Hung, and M.K. Lim, Int. J. Solids Structures 30, 3357 (1993); J. Appl. Mech. 62, 159 (1995).

34 M. C. Rigdway, C. J. Glover, G. J. Foran, and K. M. Yu, J. Appl. Phys. 83, 4610 (1998); Ingvar Ebbsjö et al., J. Appl. Phys. 87, 7708 (2000).

${ }^{35}$ W. N. Sharpe Jr., B. Yuan, R. Vaidyanathan, and R. L. Edwards, in Proceedings of the 10th IEEE International Workshop on Microelectromechanical Systems, 424 (1997).

36 A. Khan, J. Philip, and P. Hess, J. Appl. Phys. 95, 1667 (2004).

37 M. A. El Khakani, M. Chaker, M. E. OHern, and W. C. Oliver, J. Appl. Phys. 82, 4310 (1997);

R. F. Wiser, M. Tabib-Azar, M. Mehregany, and C. A. Zorman, J. Microelectromech. Syst. 14, 579 (2005).

38 D. V. Anghel and M. Manninen, Phys. Rev. B 59, 9854 (1999).

39 N.W. Ashcroft and N.D. Mermin, Solid State Physics (Saunders College, Philadelphia, 1976).

40 W. Yi, L. Lu, Z. Dian-lin, Z.W. Pan, and S.S. Xie, Phys. Rev. B 59, R9015 (1999); J. Hone, B. Batlogg, Z. Benes, A.T. Johnson, and J.E. Fischer, Science 289, 1730 (2000).

41 J.C. Lasjaunias, K. Biljakovic, Z. Benes, J.E. Fischer, and P. Monceau, Phys. Rev. B 65, 113409 (2002). 
42 B.A. Glavin, Phys. Rev. Lett. 86, 4318 (2001).

43 V.N. Popov, Phys. Rev. B 66, 153408 (2002).

44 R.C. Zeller and O. Pohl, Phys. Rev. B 4, 2029 (1971). 